\title{
A Mobile Decision Support System for Dynamic Group Decision Making Problems
}

\author{
I.J. Pérez, F.J. Cabrerizo and E. Herrera-Viedma
}

\begin{abstract}
-
The aim of this paper is to present a decision support system model with two important characteristic: (i) mobile technologies are applied in the decision process, and (ii) the set of alternatives is not fixed over time to address dynamic decision situations in which the set of solution alternatives could change throughout the decision making process. We implement a prototype of such mobile decision support system in which experts use mobile phones to provide their preferences anywhere and anytime. In order to get a general system, experts' preferences are assumed to be represented by different preference representations: fuzzy preference relations, orderings, utility functions, and multiplicative preference relations. Because this prototype incorporates both selection and consensus processes, it allows to model group decision making situations. The prototype incorporates a tool to manage the changes on the set of feasible alternatives that could happen throughout the decision process. In such a way, the prototype provides a new approach to deal with dynamic group decision making situations to help making decisions anywhere and anytime.
\end{abstract}

keywords: Group decision making, mobile internet, decision support system.

\section{INTRODUCTION}

A decision making process, consisting in deriving the best option from a feasible set, is present in just about every conceivable human task. As a result, the study of decision making is necessary and important not only in Decision Theory but also in areas such as Management Science, Operations Research, Politics, Social Psychology, Artificial Intelligence, Soft Computing, and so on.

It is obvious that the comparison of different actions according to their desirability in decision problems, in many cases, cannot be done by using a single criterion or an unique person. Thus, we interpret the decision process in the framework of group decision making (GDM) [1], [2]. This has led to numerous evaluation schemes, and has become a major concern of research in decision making. Several authors have provided interesting results on GDM with the help of fuzzy theory, and the reader is invited to see the following references [1], [3], [4], [5], [6], [7], [8], [9], [10], [11].

The central goal of decision support systems (DSS) [12], [13], [14] is to process and provide suitable information in order to support individuals or organizations in their decision making tasks. Nowadays, information can be supplied, received and or used anywhere and as such appropriate mobile

I.J. Pérez and E. Herrera-Viedma are with Dept. of Computer Science and Artificial Intelligence, University of Granada, 18071 - Granada, Spain. E-mail: ijperez@decsai.ugr.es,viedma@decsai.ugr.es

F.J. Cabrerizo is with Dept. of Software Engineering and Computer Systems, Distance Learning University of Spain (UNED), 28040 - Madrid, Spain E-mail: cabrerizo@issi.uned.es
DSSs could bridge the gap existing between theory and practice in decision making. It could also provide additional value to users, which could eventually lead to an increase in the number of successful transactions [15].

The application of the latest technologies extends opportunities in decision making, and allows to carry out consensus processes in situations that previously could not be correctly addressed. For example, nowadays it is possible to carry out consensus processes among several experts which are located in different countries around the world. However, it is important to remark that even with the adoption of mobile technologies [16], [17] there is still an important need of new collaboration and information tools for the experts being able to solve decision making problems when they cannot meet together.

In the cases where direct communication is not possible and experts do not have the possibility of gathering together, a problem arises in many consensus processes for GDM: experts may not have a clear idea about the current consensus status among all the experts involved in the decision process. In these cases, experts will probably need some assistance to establish connections among them and to obtain a clear view of the consensus process progress. This help could be provided via mobile technologies, as it can be considered a very good if not perfect way for a continuous communication flow: it allows experts to always have dynamic and updated information to determine the current consensus process status and, at the same time, it provides mechanisms to send expert preferences in real time, that is, to simulate real discussion processes. With proper DSS tools it is possible to determine which experts have similar opinions, and thus, experts may join or form different groups to better discuss every alternative, and to try to influence other experts.

The incorporation of mobile technologies in GDM processes is based on the assumption that if the communications are improved the decisions will improve, because the discussion could be focussed on the problem with less time spent on unimportant issues.

The aim of this paper is to present a prototype of mobile DSS to deal automatically with GDM problems assuming different preference representations and based on mobile technologies. This mobile DSS allows to develop dynamic GDM processes. In fact, at every stage of the decision process, the users (i) will be informed with updated data about the current stage of the decision process, (ii) will receive recommendations to help them to change their preferences, and (iii) will be able to send their updated preferences at any moment, thus improving the user participation in the GDM process. Additionally, to better simulate real decision making 
processes usually carry out in these cases, the proposed model incorporates both consensus and selection processes. Another innovation introduced in the prototype is a tool to manage not only dynamic inputs of new alternatives that, due to some dynamic external factors, can appear during the decision process, but also the outputs of some of them considered good alternatives at the beginning of the process but not so later on or are unavailable at the time. In such a way, a new approach to deal with dynamic GDM problems is presented. In order to build a flexible framework and give a high degree of freedom to represent the preferences, experts are allowed to provide their preferences in any of the following four ways: (i) as a preference ordering of the alternatives, (ii) as an utility function, (iii) as a fuzzy preference relation, or (iv) as a multiplicative preference relation.

In order to do this, the paper is set out as follows. Some general considerations about GDM models and mobile technologies are presented in Section II. Section III defines the prototype of a mobile DSS, including a practical experiment. In section IV we discuss some of its drawbacks and advantages. Finally, in Section V conclusions are drawn.

\section{PRELIMINARIES}

In this section we present the classical GDM model and the advantages of using mobile technology in GDM problems.

\section{A. Group Decision Making Models}

In a GDM problem we have a finite set of feasible alternatives, $X=\left\{x_{1}, x_{2}, \ldots, x_{n}\right\},(n \geq 2)$, to be classified from best to worst using the information given by a set of experts, $E=\left\{e_{1}, e_{2}, \ldots, e_{m}\right\},(m \geq 2)$.

Usual resolution methods for GDM problems include two different processes [18], [8] (see Figure 1):

1) Consensus process: Clearly, in any decision process, it is preferable that the experts reach a high degree of consensus on the solution set of alternatives. Thus, this process refers to how to obtain the maximum degree of consensus or agreement between the set of experts on the solution alternatives.

2) Selection process: This process consists in how to obtain the solution set of alternatives from the opinions on the alternatives given by the experts.

Usually, resolution methods for GDM problems are static, that is, it is assumed that the number of alternatives and experts acting in the GDM problem remains fixed throughout the decision making process. However, in real decision situations we find dynamic GDM problems in which the number of alternatives and/or experts could vary during the decision making process. In this paper, we assume dynamic GDM problems with possible changes on the set of alternatives.

On the other hand, as each expert, $e_{k} \in E$, has his own ideas, attitudes, motivations and personality, it is quite natural to think that different experts could express their preferences in a different way. This fact has led some authors [19], [20], [21], [22], [23], [24] to assume that experts' preferences over the set of alternatives may be represented in different ways.

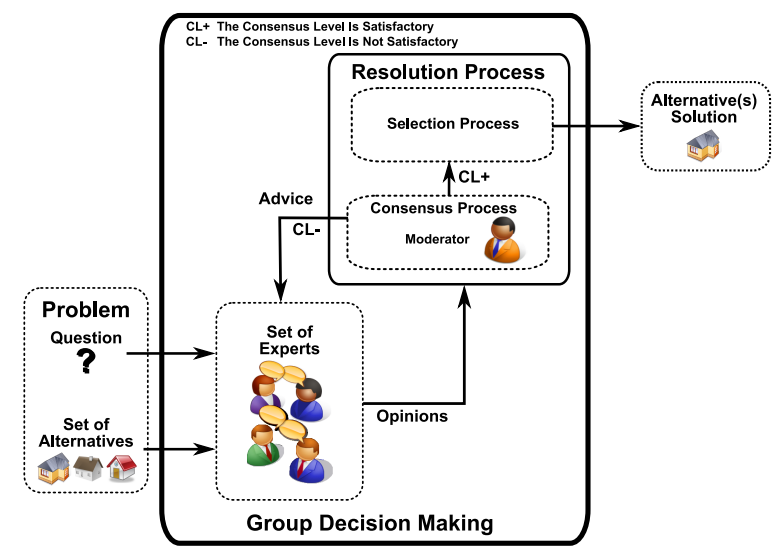

Fig. 1. Resolution process of a GDM

Amongst these, the most frequently used in decision making theory are:

- Preference orderings of alternatives: $O^{k}=$ $\left\{o^{k}(1), \ldots, o^{k}(n)\right\}$, where $o^{k}(\cdot)$ is a permutation function over the index set, $\{1, \ldots, n\}$, for the expert, $e_{k}$, defining an ordered vector of alternatives, from best to worst.

- Utility functions: $U^{k}=\left\{u_{1}^{k}, \ldots, u_{n}^{k}\right\}, u_{i}^{k} \in[0,1]$, where $u_{i}^{k}$ represents the utility evaluation given by the expert $e_{k}$ to $x_{i}$.

- Fuzzy preference relations: $P^{k} \subset X \mathrm{x} X$, with a membership function, $\mu_{P^{k}}: X \times X \rightarrow[0,1]$, where $\mu_{P^{k}}\left(x_{i}, x_{j}\right)=$ $p_{i j}^{k}$ denotes the preference degree of $x_{i}$ over $x_{j}$.

- Multiplicative preference relations: $A^{k} \subset X \times X X$, where the intensity of preference, $a_{i j}^{k}$, is measured using a ratio scale, particularly the $1 / 9$ to 9 scale.

\section{B. Mobile Technologies in GDM Problems}

In this subsection we present the advantages and limitations of new mobile technologies, and we discuss the usage of mobile devices to solve GDM problems.

1) Advantages and limitations: Mobile communication systems are characterized by a variety of features [16], [17]. They differ from each other in the degree of their complexity, the level of the offered services and their operational costs.

The Mobile Web refers to the World Wide Web accessed from mobile devices such as cell phones, PDAs, and other portable gadgets connected to a network. So, access to web services no longer requires a desktop computer. The following list shows the different advantages that mobile technologies can provide [16], [17]:

- Internet has provided an easy and effective way of delivering information and services to millions of users who are connected to wired network. Evidently, this wired network addresses two major constraints: time and place. These limitations have raised the issue of the mobile internet, which enables users to access information from any place at any moment using a mobile wireless device. The possibility to have access to this kind of services in wireless environments provides a great mobility to the users. This mobility can increase the productivity due to 
the increasing agility of some tasks, can allow to save displacements and infrastructure's costs, can improve business processes, can ease decision making processes obtaining more dynamic and precise solutions, and can even improve the offered services.

- The mobile computing paradigm has several interesting and important applications for business, telecommunications, real-time control systems and remote operations [15], [25], [26].

- Recently, the fast technological innovation made it possible to provide secure, fast and quality communications through the wireless network. Moreover, devices that used to deliver limited information are now able to provide a wide range of information and services such as e-mail, banking, entertainment and even games.

However, Mobile Web access today still suffers from some interoperability and usability problems. This is partly due to the small physical size of the screens of mobile devices and partly due to the incompatibility of many mobile devices with both computer operating systems and the format of much of the available information on the Internet.

Some of the limitations that current mobile services have to face are:

- Small screen size: It is difficult or impossible to properly adapt text and graphics prepared for the standard size of a desktop computer screen with current information standards.

- Lack of windows: On mobile web only one page can be displayed at a time, and pages usually can only be viewed in the sequence they were originally accessed.

- Navigation: Usual mobile devices do not use a mouse like pointer, but rather simply an up and down function for scrolling, thereby limiting the flexibility of navigation.

- Format of accessible pages: Many sites that can be accessed on a desktop cannot on a mobile device. Many devices cannot show pages with a secured connection, Flash or other similar elements, PDFs, or video sites.

- Speed: On most mobile devices, the speed of service is very slow, often slower than dial-up internet access.

- Size of messages: Many devices have limits on the number of characters that can be sent in a single message.

To make use of mobile technology in the best way, several conditions need to be fulfilled. The first condition, nowadays achieved, is the widespread use of mobile devices that connect individuals to the mobile network and the contents that provide useful information and services to users. In addition, the technological support in terms of speed, communication quality and security are also important in the development of the mobile technology [13].

The mobile Web mainly uses lightweight pages written in Extensible Hypertext (XHTML) or Wireless Markup Language (WML), to deliver content to mobile devices. However, new tools such as Macromedia's Flash Lite or Sun's J2ME enable the production of richer user interfaces customized for mobile devices.

2) Usage of Mobile Technology in GDM Problems: During the last decade, organizations have moved from face-to-face group environments to virtual group environments using communication technology. More and more workers use mobile devices to coordinate and share information with other people. The main objective is that the members of the group could work in an ideal way where they are, having all the necessary information to take the right decisions [16], [17], [27], [28].

To support the new generation of decision makers and to add real-time process in the GDM problem field, many authors have proposed to develop decision support systems based on mobile technologies [29], [30]. Similarly, we propose to incorporate mobile technologies in a DSS obtaining a Mobile DSS (MDSS). Using such a technology should enable a user to maximize the advantages and minimize the drawbacks of DSSs.

The need of a face-to-face meeting disappears with the use of this model, being the own computer system who acts as moderator. Experts can communicate with the system directly using their mobile device from any place in the world and at any time. Hereby, a continuous information flow among the system and each member of the group is produced, which can help to reach the consensus between the experts on a faster way and to obtain better decisions.

In addition, MDSS can help to reduce the time constraint in the decision process. Thus, the time saved by using the MDSS can be used to do an exhaustive analysis of the problem and obtain a better problem definition. This time also could be used to identify more feasible alternative solutions to the problem, and thus, the evaluation of a large set of alternatives would increase the possibility of finding a better solution. The MDSS helps to the resolution of GDM problems providing a propitious environment for the communication, increasing the satisfaction of the user and, in this way, improving the final decisions.

\section{A MOBILE Decision SUPPORT SySTEM BASED ON DYNAMIC CHOICE OF ALTERNATIVES}

While DSSs have typically been associated with desktop systems and involve considerable processing, the development of new compact and mobile technologies provides new opportunities to develop this kind of DSSs over mobile Internet (M-Internet) [12], [16], [17].

In this section we describe the implemented GDM model that incorporates a tool to manage dynamic decision models in which the alternatives of the set of solution alternatives could change throughout decision process and uses different formats to represent preferences. It allows to develop GDM processes at anytime and anywhere, and simulate with more accuracy level the real processes of human decision making which are developed in dynamic environments as the Web, financial investment, health, etc. Finally, the prototype of this mobile DSS is presented.

\section{A. Structure of the Implemented GDM Model}

The structure of the proposed Mobile DSS model is composed of the following five processes: (i) uniformization process, (ii) selection process, (iii) consensus process, (iv) dynamic choice process of alternatives, and (v) feedback process (Figure 2). 


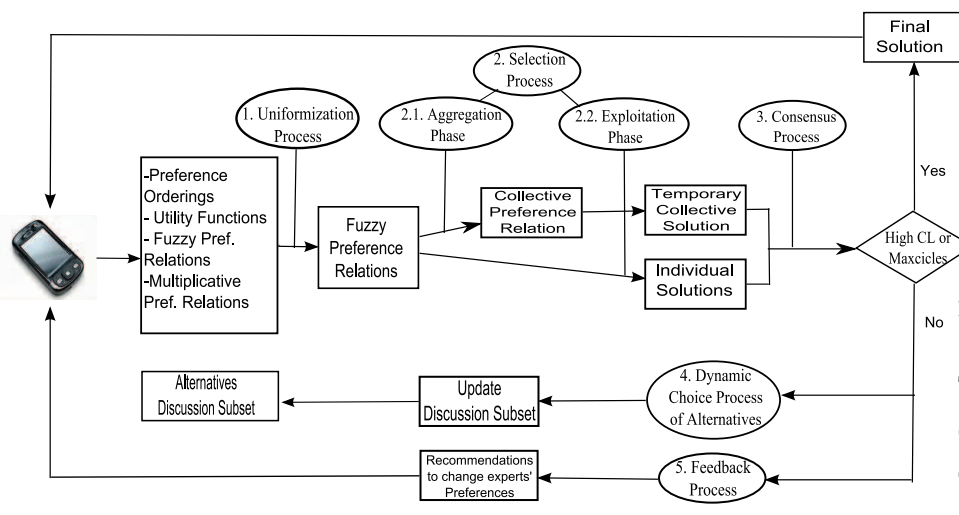

Fig. 2. Operation of the GDM model with multiple preference representation structures

1) Uniformization Process: To give a higher degree of freedom to the system, we assume that experts can present their preferences using any of the preference representations presented in section II-A. Therefore, it is necessary to make the information uniform before applying the consensus and selection processes. As in [20] we propose to use fuzzy preference relations as the base element to uniform experts' preferences and the following transformation functions are used [20]: $f^{1}\left(o_{i}^{k}, o_{j}^{k}\right)=\frac{1}{2}\left(1+\frac{o_{j}^{k}-o_{i}^{k}}{n-1}\right), f^{2}\left(u_{i}^{k}, u_{j}^{k}\right)=$ $\frac{\left(u_{i}^{k}\right)^{2}}{\left(u_{i}^{k}\right)^{2}+\left(u_{j}^{k}\right)^{2}}, f^{3}\left(a_{i j}^{k}\right)=\frac{1}{2}\left(1+\log _{9} a_{i j}^{k}\right)$.

2) Selection Process: Once the information is made uniform, we have a set of $m$ individual fuzzy preference relations and then we apply a selection process which has two phases [2], [31]: (i) aggregation and (ii) exploitation.

\section{- Aggregation phase:}

This phase defines a collective preference relation, $P^{c}=$ $\left(p_{i j}^{c}\right)$, obtained by means of the aggregation of all individual fuzzy preference relations $\left\{P^{1}, P^{2}, \ldots, P^{m}\right\}$. It indicates the global preference between every pair of alternatives according to the majority of experts' opinions. For example, the aggregation could be carried out by means of an OWA operator [32], [33].

\section{- Exploitation phase:}

This phase transforms the global information about the alternatives into a global ranking of them, from which the set of solution alternatives is obtained. The global ranking is obtained applying two choice degrees of alternatives to the collective fuzzy preference relation [7]: the quantifier guided dominance degree (QGDD) and the quantifier guided non dominance degree (QGNDD).

Finally, the solution $X_{\text {sol }}$ is obtained by applying these two choice degrees, and thus, selecting the alternatives with maximum choice degrees.

3) Consensus Process: In our mobile DSS, we use a consensus model for GDM problems with different preference representations as it was done in [34]. This model presents the following main characteristics:

- It is based on two soft consensus criteria: global consensus measure on the set of alternatives $X$, symbolized as $C_{X}$, and the proximity measures of each expert $e_{i}$ on $X$, called $P_{X}^{i}$.

- Both consensus criteria are defined by comparing the individual solutions with the collective solution using as comparison criterion the positions of the alternatives in each solution.

Initially, in this consensus model we consider that in any nontrivial GDM problem the experts disagree in their opinions so that consensus has to be viewed as an iterated process. This means that agreement is obtained only after some rounds of consultation. In each round, the DSS calculates both the consensus and the proximity measures. The consensus measures evaluate the agreement existing among experts and the proximity measures are used in the feedback mechanism to support the group discussion phase of the consensus process.

4) Dynamic Choice Process of Alternatives: In real world we find many dynamic decision frameworks: health, financial investment, military operations, Web. In such cases, due to different factors the set of solution alternatives could vary throughout the decision process. A typical example of this situation is the medical diagnosis. This environment is dynamic in the sense that a patient could present new symptoms or he could set better due to the medication, and thus, any change of state of the patient should be taken into account by the doctors.

Classical GDM models are defined within static frameworks. In order to make the decision making process more realistic, we provide a new tool to deal with dynamic alternatives in decision making. In such a way, we can solve dynamic decision problems in which, at every stage of the process, the discussion could be centered on different alternatives.

To do so, we define a method which allows us to remove and insert new alternatives into the discussion process. Firstly, the system identifies those worst alternatives that might be removed and the new alternatives to include in the set. This new alternatives can be obtained from a set of new alternatives appeared at a time or from the supply set of alternatives that includes all the alternatives that we had at the beginning of the process but that were not included in the discussion subset because the limitation of this due to specific parameters of the problem.

Thus, the method has two different phases: (1) Remove old bad alternatives and and (2) Insert new good alternatives.

1) The first phase manages situations in which some alternatives of the discussion subset are not available at the moment due to some dynamic external factors or because the experts have evaluated them poorly and they have a low dominance degree $(Q G D D)$. Therefore, the system checks the availability and the $Q G D D$ of each alternative in the current discussion subset. If some alternative is not available or has a $Q G D D$ lower than a threshold $(\min Q G D D)$, the system looks for a new good alternative in the new alternatives subset. If this subset is empty, the system uses the supply subset of alternatives provided by the expert at the beginning of the decision process and that were not taken into account then because of the impossibility to compare all the alternatives at the same time. Then, the system asks for the experts' 
opinions about the replacement and acts according to them (see Figure 3).

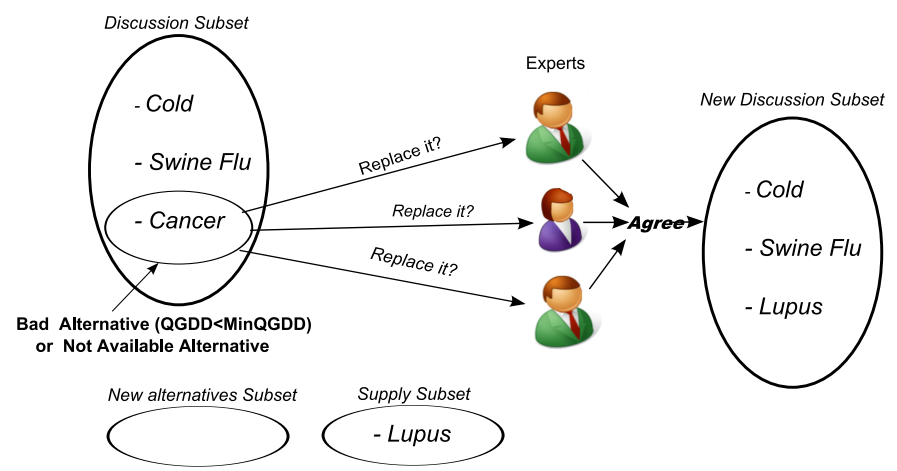

Fig. 3. Dynamic choice process of alternatives: Case 1

2) The second case manages the opposite situation, that is, when some new alternatives have emerged. Basically, the system checks if some new good alternatives have appeared in the new alternatives subset due to some dynamic external factors. If this is the case, the system has to identify the worst alternatives of the current discussion subset. To do this, the system uses the dominance degree $Q G D D$ of all alternatives again to choose the worst alternatives. Then, the system asks for the experts' opinions about the replacement and acts according to them (see Figure 4).

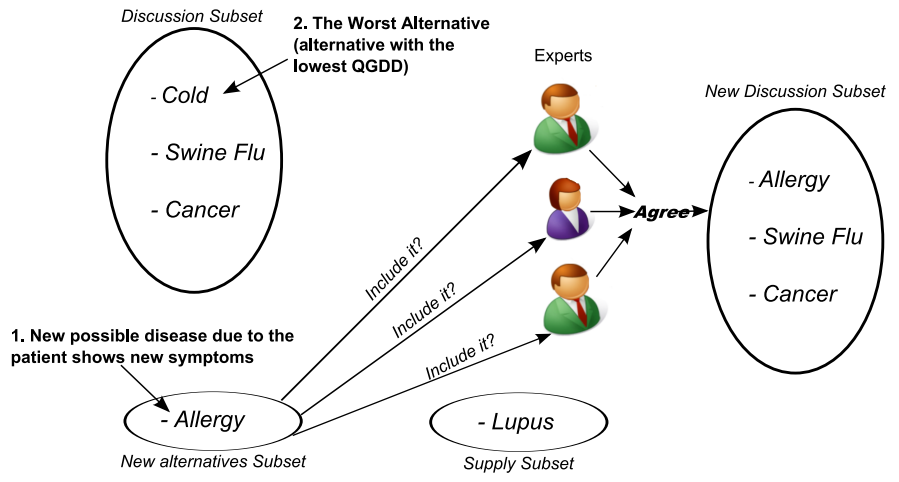

Fig. 4. Dynamic choice process of alternatives: Case 2

To avoid stagnation at this point a maxTime threshold is established. If the majority of experts that answered the question in maxTime think that the changes are appropriate, the system updates the discussion subset according to the above cases. The possibility of these changes makes experts to be more involved in the process and improve their satisfaction with the final results.

5) Feedback Process: To guide the change of the experts' opinions, the DSS simulates a group discussion session in which a feedback mechanism is applied to quickly obtain a high level of consensus. This mechanism is able to substitute the moderator's actions in the consensus reaching process. The main problem is how to find a way of making individual positions converge and, therefore, how to support the experts in obtaining and agreeing with a particular solution.
When the consensus measure $C_{X}$ has not reached the required consensus level (CL) and the number of rounds has not reached a maximum number of iterations (MAXCYCLE), defined prior to the beginning of the decision process, the experts' opinions must be modified. As aforementioned, we are using the proximity measures to build a feedback mechanism so that experts can change their opinions and narrow their positions.

This feedback mechanism uses the proximity measures to give simple rules on how to change experts' preferences.

- Rules to change the preferences:

The rules provided by the feedback mechanism are easy to understand and apply because they are provided in a natural language:

1) Each expert $e_{i}$ is classified by associating them to their respective total proximity measure $P_{X}^{i}$. Each expert is given his position and his proximity in each alternative.

2) If the expert's position in the ranking is high (first, second, etc.) then that expert should not change his opinion much, but if it is low, then that expert has to change his opinion substantially. In other words, the experts to change their opinions are those whose individual solutions are furthest from the collective temporary solution. At this point, we have to calculate how many experts have to change their opinions using a threshold defined at the beginning of the decision process.

Then the rules to change opinions are the following:

- If proximity of alternative $p_{i}\left(x_{j}\right)$ is positive then we have the rule: "Decrease values associated to alternative $x_{j}$ "

- If proximity of alternative $p_{i}\left(x_{j}\right)$ is negative then we have the rule: "Increase values associated to alternative $x_{j}$.

\section{B. Prototype of the Mobile DSS}

Here we present the prototype of the mobile DSS, explaining the architecture of the system and the communication and work flow that summarizes the functions of the DSS .

A DSS can be built in several ways, and the used technology determines how a DSS has to be developed [14], [15]. The chosen architecture for our prototype of Mobile DSS, is a "Client/Server" architecture, where the client is a mobile device. The client/server paradigm is founded on the concept that clients (such as personal computers, or mobile devices) and servers (computer) are both connected by a network enabling servers to provide different services for the clients. Furthermore, the technologies that we have used to implement the prototype of the Mobile DSS comprise Java and Java Midlets for the client software, PHP for the server functions and MySQL for the database management.

According to the GDM model proposed in the previous section, the prototype lets the user send his/her preferences to the DSS by means of a mobile device, and the system returns to the expert the final solution or recommendations to increase the consensus level, depending on the stage of the decision 
process. An important aspect is that the user-system interaction can be done anytime and anywhere which facilitates expert's participation and the resolution of the decision process.

In what follows, we describe in detail the client and server of the Mobile DSS prototype.

1) Client: For the implementation of the DSS we have chosen a thin client model. This model depends primarily on the central server for the processing activities. This prototype is designed to operate on mobile devices with Internet connection.

The client software has to show to the experts the next eight interfaces:

- Connection: The device must be connected to the network to send/receive information to the server.

- Authentication: The device will ask for a user and password data to access the system (see Figure 5).
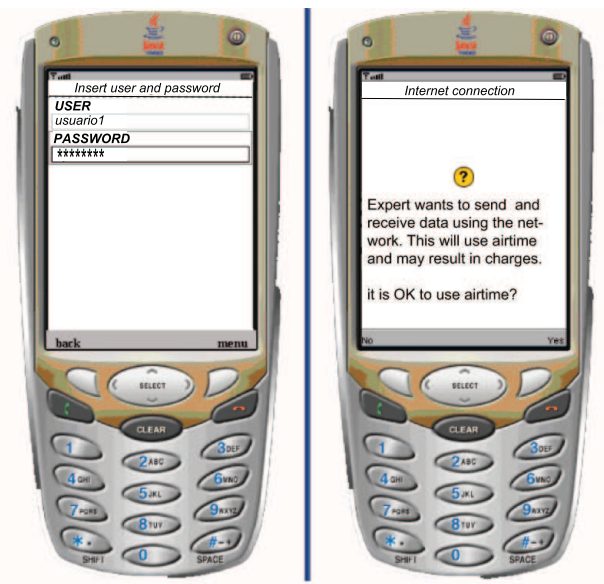

Fig. 5. Authentication and mobile Internet connection

- Problem description: When a decision process is started, the device shows to the experts a brief description of the problem and the discussion subset of alternatives (see Figure 6 a).

- Selection of preference representations (see Figure $6 \mathrm{~b}$ ).
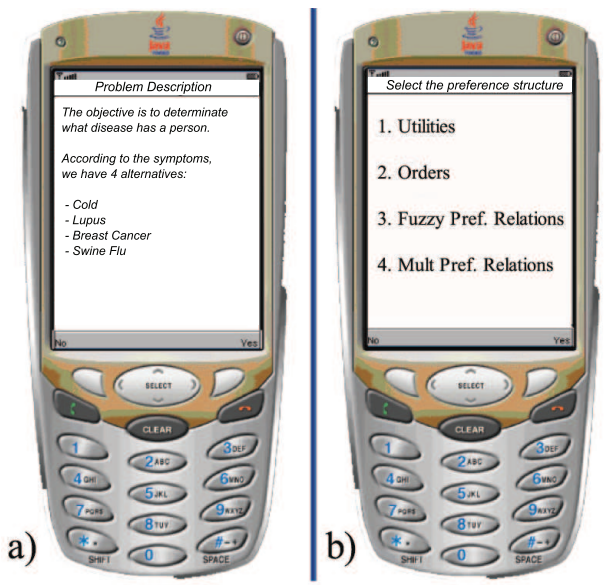

Fig. 6. Problem description and selection of preference representations

- Insertion of preferences: The device will have four different interfaces, one for each different format of preference representation (see Figure 7).

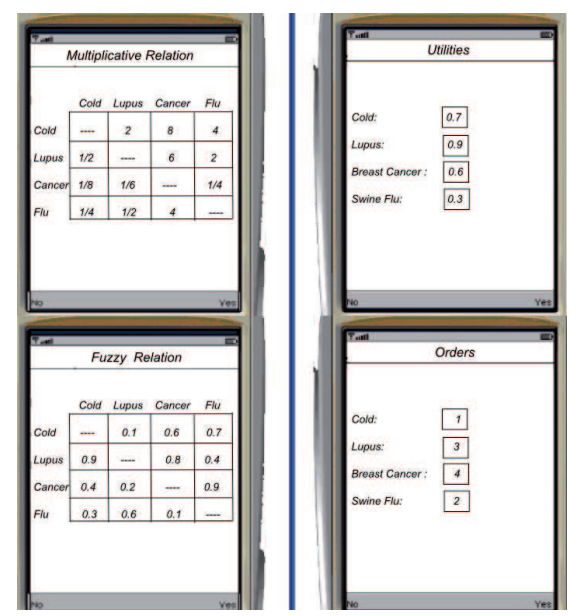

Fig. 7. Insertion of preferences

- Change of alternatives: When a bad or not available alternative deserves to be removed from the discussion subset, or a new alternative deserves be inserted in the discussion subset, using the new management process of alternatives, the experts can assess if they want to update the discussion subset by changing these alternatives (see Figure 8).

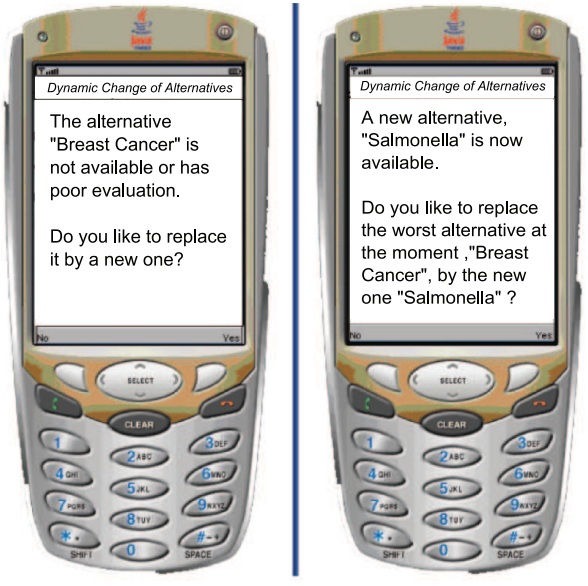

Fig. 8. Change of alternatives question

- Feedback: When opinions should be modified, the device shows to the experts the recommendations and lets them send their new preferences (see Figure 9 a).

- Output: At the end of the decision process, the device will show to the experts the set of solution alternatives as an ordered set of alternatives marking the most relevant ones (see Figure $9 \mathrm{~b}$ ).

On the technical side of the development of the client part of the DSS, it is worth noting that the client application complies with the MIDP 2.0 specifications [35], and that the J2ME Wireless Toolkit 2.2 [36] provided by SUN was used in the development phase. This wireless toolkit is a set of tools that provide J2ME developers with some emulation environments, documentation, and examples to develop MIDP-compliant 


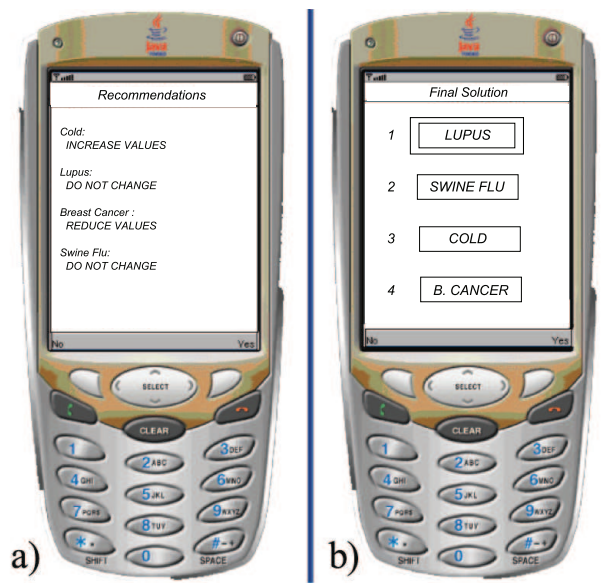

Fig. 9. Recommendations and Final Solution

applications. The application was later tested using a JAVAenabled mobile phone on a GSM network using a GPRSenabled SIM card. The MIDP application is packaged inside a JAVA archive (JAR) file, which contains the applications classes and resource files. This JAR file is the one that actually is downloaded to the physical device (mobile phone) along with the JAVA application descriptor file when an expert wants to use the MDSS.

2) Server: The server is the other fundamental part of the DSS. It is based on five main modules, which receive/send information from/to the experts through M-Internet technologies (see Figure 10).

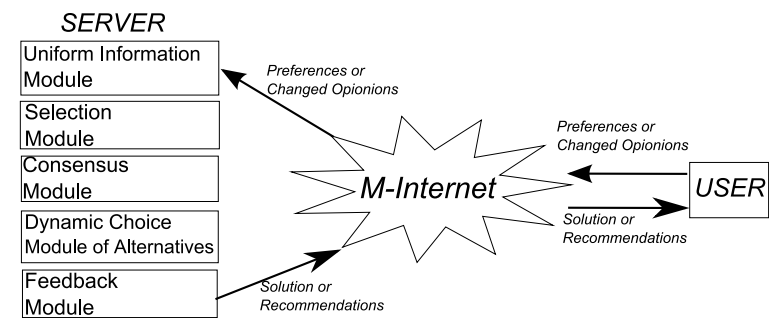

Fig. 10. Operation structure of Mobile DSS prototype

- An Uniform Information Module: This module make expert preferences uniform by using the transformatio functions presented in section III-A1, to convert all dit ferent types of preferences into fuzzy preference relation:

- A Selection Module: Once the information is made un form, the server applies the selection process to obtain temporary solution of the problem. This process has tw phases: Aggregation and Exploitation. In the aggregatio phase, the collective fuzzy preference relation is obtainec In the exploitation phase, the server obtains the quantifie guided dominance degrees of alternatives acting over th collective fuzzy preference relation. This degrees allow to establish an order in the alternatives to obtain the ranking of the temporary alternative solutions, from best to worse.

- A Consensus Module: In this module the consensus and proximity measures are calculated by the server. If the consensus measure has reached the minimum consensus level defined as a parameter of the problem, the consensus process stops, this temporary collective solution becomes the final consensual solution and is sent to the experts. In other case, the consensus process should continue.

- A Dynamic Choice Module of Alternatives: If some old alternative has to be removed from the discussion subset or some new alternative deserves to be inserted in the discussion subset, and the minimum consensus level has not been reached, the server applies the management process of alternatives to determine if the replacement should be done. To do that, the server asks the experts if they agree with the proposed change. If the majority of the experts accept it, the discussion subset of alternatives is updated by changing the worst alternative of the set by the new one or by the first one in the supply list.

- A Feedback Module: When a consensus stage is finished without reaching the minimum consensus level, the server starts a feedback mechanism that generates recommendations rules. These recommendations demand the experts to change their preferences and explain how they have to do it (increasing or decreasing some preferences).

In this way, the consensus process will converge and, eventually, the solution will reach a high consensus degree.

The server also implements a database that stores all the data of the problem as well as the experts data, alternatives data, preferences, consensus measures, recommendations, consensus parameters, selection parameters and so on.

3) Communication and work flow: The DSS has to carry out the following functions, also represented in figure 11. In the diagram we can see all the functions of the system, the form in which they are connected together with the database, and the order in which each of them is executed.

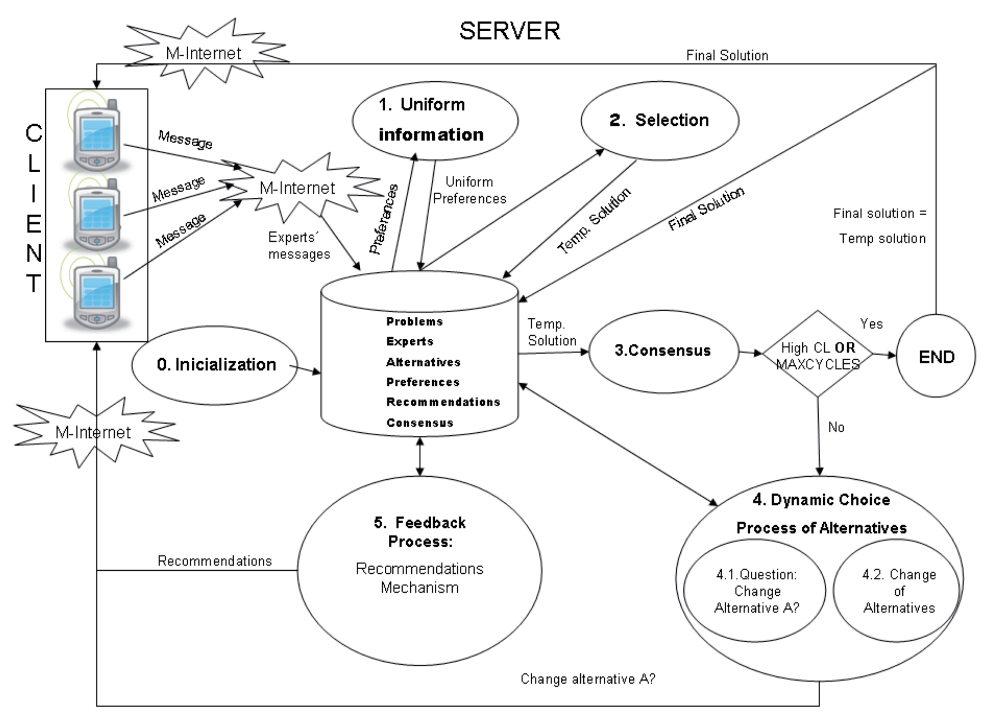

Fig. 11. Functions Scheme of the System

0) Initialization: A first step to the start of the execution of the system consist of the insertion in the database of all the initial parameters of the problem, the experts and the set of alternatives. Before starting the decision 
process is necessary to set suitable values for all of the parameters according to the problem, especially those that limit the time to be spent in its resolution. It is not the same an urgent medical situation where experts have to decide the best medical treatment quickly that to choose a country to visit during holidays. In the first case, the maximum number of iterations of the consensus process and the maximum time of waiting for the expert opinions should be shorter than the second one because the final solution is required as soon as possible. So, these values are very dependent on the problem at hand, and they have to be established according to the special needs of each situation.

1) Verify the user messages and store the main information: When an expert wants to access the system, he/she has to send a message through M-Internet using his mobile device. The user can send two kinds of messages: i) Preferences message: It is composed of authentication information (login and password) and his preferences about the problem, using any of these four available formats: preference orderings, utility functions, fuzzy preference relations or multiplicative preference relations.

ii) Change of alternatives message: It is composed by authentication information (login and password) and the answer to the change of alternatives question.

The message is verified by the server, that checks the login and password in the database. If the authentication process is correct, the rest of the information of the message is stored in the database, and the server decides when the consensus stage can start (if all experts have provided their preferences) or, when the change of alternatives mechanism can be finished (if enough experts answer the change of alternatives question).

2) Make the experts' preferences uniform: The server makes the information uniform using fuzzy preference relations as the base element of preferences representation. The server saves this information in the database.

3) Computation of the set of solution alternatives: The selection module returns the solution set of alternatives in each stage of the decision process. All the information about the temporary solution is saved in the database.

4) Computation of the consensus measures: In this step, the consensus and proximity measures are computed by the server and saved in the database.

5) Control the consensus state: In this step, the server determines if the required agreement degree has been reached (and thus, the decision process must finish by applying the selection process) or if a new round of consensus using the feedback mechanism that generates recommendations to change the experts' preferences should begin.

6) Control the change of alternatives: When the minimum consensus level has not been reached and some alternatives deserve to be removed or inserted in the discussion subset, the system offers the possibility to update the discussion subset on time.

7) Generate the recommendations: In this step, the server generates the recommendations and sends a message to the experts advising that they can use the software again for reading the recommendations and start a new consensus stage. In order to avoid that the collective solution does not converge after several discussion rounds, the prototype stops if the number of rounds reaches MAXCYCLES.

The results are saved in the database and are sent to the experts through M-Internet to help them to change their preferences.

8) Go to step 1: A new round of the decision making process starts.

The system operation will be illustrated in more detail in the next subsection with a practical example.

\section{Practical Example of the Mobile Decision Support System}

In this subsection, we are going to illustrate a simple real example of use of the DSS. It is worth noting the behavior of the system under complex problems because the prototype allows dynamic sets of alternatives because it manages their inputs and outputs in real time, and because it is able to address problems with large sets of alternatives them as well. When all the alternatives cannot be displayed on a mobile screen at the same time, the remaining ones can be ordered in a supply list and be evaluated later in the process. Therefore, the system can support a big number of experts and alternatives in order to solve complex problems. To illustrate how the prototype works, we will follow the communication flow presented in the previous section.

The experiment dealt with the choice of the best restaurant for a Christmas dinner by four members (experts) of a work group. They used their last generation mobile devices because they live in different countries and cannot gather together to plan the meeting.

At the beginning, the secretary of the work group had to look for a set of available restaurants. Later, a list of six of these available restaurants was created as the feasible candidates to celebrate the dinner. These candidates, arranged according to prize, made up the initial set of alternatives for the problem.

The first step to solve a problem using our prototype is to insert all the parameters of the problem (experts, alternatives, thresholds, timing...) in the database. (See table I, table II and table III)

\begin{tabular}{|c|c|c|c|c|}
\hline Code & Name & Capacity & Prize & City \\
\hline$r_{1}$ & Las Tinajas & 75 & 20-50 Euros & Granada \\
\hline$r_{2}$ & La Pataleta & 45 & 20-40 Euros & Granada \\
\hline$r_{3}$ & La Ermita & 55 & 22-35 Euros & Granada \\
\hline$r_{4}$ & Kudam & 60 & 25-55 Euros & Granada \\
\hline$r_{5}$ & Casa Ramon & 60 & 30-52 Euros & Granada \\
\hline$r_{6}$ & Il Gondoliere & 45 & 31-41 Euros & Granada \\
\hline
\end{tabular}

TABLE I

ALternAtives OF THE PROBLEM

When the initial parameters were defined according to the problem requirements, the decision making process started. 


\begin{tabular}{|c|c|c|c|}
\hline Code & Name & City & MobileDevice \\
\hline$e_{1}$ & Enrique & Granada (Spain) & Nokia N70 \\
\hline$e_{2}$ & Paco & Leicester (UK) & Nokia 6234 \\
\hline$e_{3}$ & Javier & Madrid (Spain) & HTC Touch \\
\hline$e_{4}$ & Sergio & Granada (Spain) & LG Viewty \\
\hline
\end{tabular}

TABLE II

EXPERTS OF THE PROBLEM AND MOBILE DEVICES USED

\begin{tabular}{|c|c|c|}
\hline Name & Value & Description \\
\hline$b$ & 1 & Control the proximity measures \\
\hline$\beta$ & 0.5 & Control the S-OWA operator \\
\hline minConsDegree & 0.8 & Minimum consensus level \\
\hline minProxDegree & 0.7 & Minimum proximity level \\
\hline MAXCYCLES & 4 & Maximum number of iterations \\
\hline maxTime & 12 (hours) & Maximum waiting time \\
\hline minQGDD & 0.2 & Minimum dominance level \\
\hline DSsize & 4 & Discussion subset size \\
\hline
\end{tabular}

TABLE III

INITIAL PARAMETERS OF THE PROBLEM

Note that the set of alternatives has six restaurants $X=$ $\left\{R_{1}, \ldots, R_{6}\right\}$ but we suppose that the experts are not able to compare all of them altogether. Thus, they will evaluate only four of them (DSsize $=4)$, that is, the initial discussion subset will consist on the first four, $X^{\prime}=\left\{R_{1}, \ldots, R_{4}\right\}$. The remaining restaurants are included in the supply set to support some changes in the discussion subset at the following iterations of the decision process. These changes could be made when some of the current restaurants obtain a low evaluation or are not available for booking anymore.

The first four restaurants are presented to the group of four experts, $E=\left\{e_{1}, \ldots, e_{4}\right\}$. They are asked to give their opinions about them using our mobile decision support system.

The experiment was carried out using a real set of lastest technology mobile devices (see table II). Therefore we have to illustrate the input and output interfaces using a mobile emulator provided by "Sun Microsystem". The input and output data sets are the same that in the real experiment. The interfaces depend on the device screen but are very similar.

Expert $e_{1}$ gave his opinions using preference orderings, $e_{2}$ using utility values, $e_{3}$ using fuzzy preference relations and finally, $e_{4}$ using multiplicative preference relations. Experts' initial opinions are shown in Figure 12.

These preferences and the authentication information are sent to the server by each expert and, if the authentication process is correct, the preferences are stored in the table preferences of the database. When the last expert has sent his message, the decision process is started by the server.

1) First stage in the decision process:

a) Uniform information module: Using the transformation functions presented in section III-A, the system obtains the following individual fuzzy preference relations:

$$
P^{1}=\left(\begin{array}{cccc}
0.5 & 0.16 & 0.33 & 0 \\
0.83 & 0.5 & 0.66 & 0.33 \\
0.66 & 0.33 & 0.5 & 0.16 \\
1 & 0.66 & 0.83 & 0.5
\end{array}\right)
$$

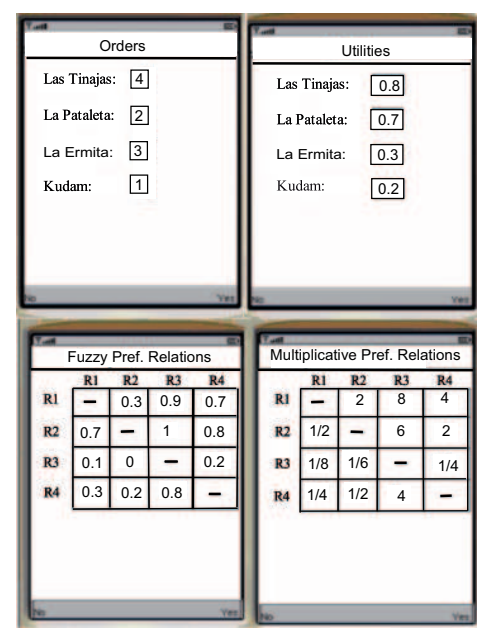

Fig. 12. Expert preferences

$$
\begin{aligned}
P^{2} & =\left(\begin{array}{cccc}
0.5 & 0.57 & 0.88 & 0.94 \\
0.43 & 0.5 & 0.84 & 0.92 \\
0.22 & 0.16 & 0.5 & 0.69 \\
0.06 & 0.08 & 0.21 & 0.5
\end{array}\right) \\
P^{3} & =\left(\begin{array}{cccc}
0.5 & 0.3 & 0.9 & 0.7 \\
0.7 & 0.5 & 1 & 0.8 \\
0.1 & 0 & 0.5 & 0.2 \\
0.3 & 0.2 & 0.8 & 0.5
\end{array}\right) \\
P^{4} & =\left(\begin{array}{cccc}
0.5 & 0.66 & 0.97 & 0.82 \\
0.34 & 0.5 & 0.91 & 0.66 \\
0.03 & 0.09 & 0.5 & 0.18 \\
0.18 & 0.34 & 0.82 & 0.5
\end{array}\right)
\end{aligned}
$$

These four relations are also stored in the table preferences of the database.

b) Selection module: Using the fuzzy majority criterion with the corresponding OWA operator with the weighting vector $W=[0.5,0.2,0.17,0.13]$ ("most of"), the collective fuzzy preference relation is computed.

$$
P^{c}=\left(\begin{array}{cccc}
0.5 & 0.52 & 0.86 & 0.75 \\
0.48 & 0.5 & 0.91 & 0.77 \\
0.14 & 0.09 & 0.5 & 0.44 \\
0.25 & 0.23 & 0.56 & 0.5
\end{array}\right)
$$

We apply the exploitation process with the corresponding OWA operator with the weighting vector $W=$ $[0.07,0.67,0.26]$ ("most of"), and compute the dominance choice degree $\left(Q G D D_{i}\right)$ over the collective fuzzy preference relation: $Q G D D_{1}=0.696, Q G D D_{2}=0.702, Q G D D_{3}=$ $0.146, Q G D D_{4}=0.265$.

These values represent the dominance that one alternative has over "most of" the alternatives according to "most of" the experts.

We can see that the best current candidate is $R_{2}$, and the collective order of restaurants is $\left\{R_{2}, R_{1}, R_{4}, R_{3}\right\}$. This order can be seen as our temporary solution in this first consensus stage. 
c) Consensus module: The system computes the individual orders for each expert in a similar way to the global solution:

$$
\begin{array}{ll}
e_{1}: & \left\{R_{4}, R_{2}, R_{3}, R_{1}\right\} \\
e_{2}: & \left\{R_{1}, R_{2}, R_{3}, R_{4}\right\} \\
e_{3}: & \left\{R_{1}, R_{2}, R_{4}, R_{3}\right\} \\
e_{4}: & \left\{R_{2}, R_{1}, R_{4}, R_{3}\right\}
\end{array}
$$

Consensus degrees of the set of experts over the individual alternatives are: $C\left(R_{1}\right)=0.55, C\left(R_{2}\right)=0.66, C\left(R_{3}\right)=$ $0.77, C\left(R_{4}\right)=0.66$.

The global consensus measure is computed using an OWA operator and we obtain the following: $C_{X}=0.67$.

The proximity measures are also computed using an OWA operator: $P_{X}^{1}=0.55, P_{X}^{2}=0.67, P_{X}^{3}=0.78, P_{X}^{4}=1$.

As we can see, the consensus has not reached the minimum required by the problem $\left(C_{X}<0.8\right)$ and consequently, the decision process should continue applying both the dynamic choice process of alternatives and the feedback process.

d) Dynamic choice process of alternatives: As soon as the system has verified that the minimum consensus level amongst the experts has not been reached and before beginning a new round of consensus, it is necessary to update all the information of the problem that could be changed during the process.

To do so, the system tries to remove and replace the restaurants that cannot be booked at the moment due to their been already fully booked or whose dominace degree is below the required minimum value, i.e. $Q G D D_{i}<\operatorname{Min} Q G D D=0.2$. New restaurants or restaurants in waiting in the supply list are given as replacement alternatives. In this case, all the restaurants are available for booking, however restaurant "La Ermita" has a choice degree $Q G D D_{3}$ lower than $M i n Q G D D$. Anyway, due to external factors, bookings cancelled, a new good restaurant called "Rodizio" is now available to celebrate diner. So, the list of new alternatives has a new element and the system suggests to remove the bad restaurant and insert the new one in the discussion subset.

\begin{tabular}{|c|c|c|c|c|}
\hline Code & Name & Capacity & Prize & City \\
\hline$r_{7}$ & Rodizio & 50 & 30-50 Euros & Granada \\
\hline
\end{tabular}

TABLE IV

New Alternative of THE PROBlem

Because there are not any more new alternatives, the question (Figure 13) is sent to all the experts and the system waits for the experts' answers to update the discussion subset. Experts $e_{1}, e_{3}$ and $e_{4}$ answer that they agree with the change. $e_{2}$ does not answer the question within the threshold waiting time maxTime. Thus, the restaurant $R_{3}$ is replaced with the new restaurant, $R_{7}$, into the discussion subset of alternatives.

e) Feedback process: Next, the feedback process is applied and recommendation to the experts are given on their preference values to change in order to improve the consensus level. This is done in the following two steps:

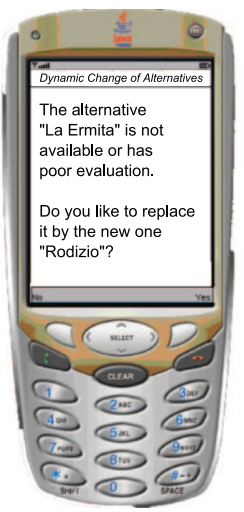

Fig. 13. Change of Alternative Question

- Classification of experts: The system ranks the experts according to their proximity measures: $e_{4}, e_{3}, e_{2}, e_{1}$.

- Changing the opinions: At this point, two of the experts, $e_{1}$ and $e_{2}$, whose proximity measures are lower than the parameter minProxDegree, are asked to change their opinions. They are not requested to change preferences on the restaurant $R_{3}$ because is replaced by $R_{7}$. Obviously, all the experts were asked to introduce their preferences about the new alternative $R_{7}$.

We can see the recommendations received by the experts in their mobile devices in Figure 14.

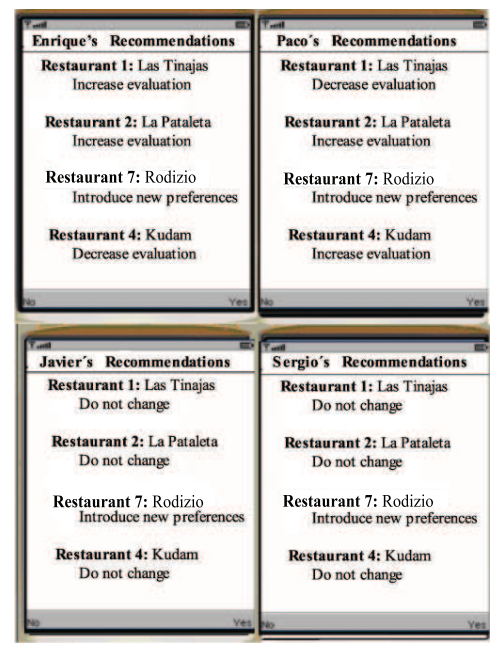

Fig. 14. Recommendations

2) Second stage in the decision process: In this stage all the experts have to send their preferences again, because the alternative set has been modified (the candidate $R_{7}$ replaced the candidate $R_{3}$ ). Experts $e_{1}$ and $e_{2}$ also received recommendations to change their preferences because their proximity levels were low in the previous round.

The experts' opinions given in the second round are shown in Figure 15.

The uniform information module transforms these preferences to fuzzy preference relations, and the selection module, with the same operations that in the previous stage, obtains a new temporary solution. The new collective ranking of restaurants is: $\left\{R_{2}, R_{1}, R_{7}, R_{4}\right\}$. 


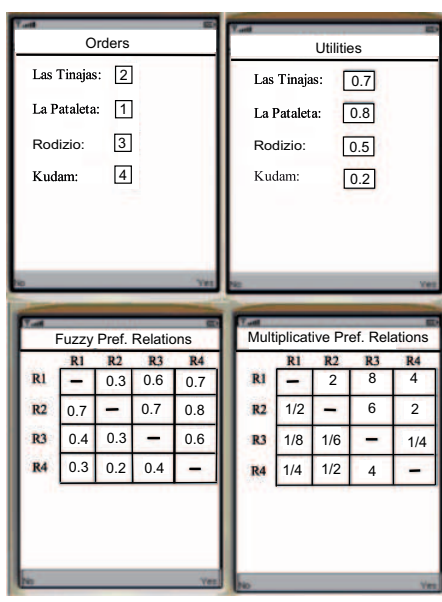

Fig. 15. New experts' preferences

The next module, "Consensus module”, obtains the consensus level: $C_{X}=0.88$.

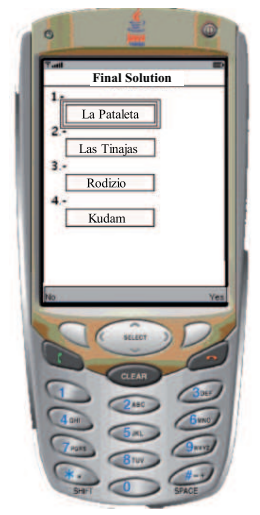

Fig. 16. Final solution

This consensus level has reached the minimum level required by the problem $\left(C_{X}>0.8\right)$, in this case the decision making process has finished, being $R_{2}$ the best alternative. The restaurants $R_{1}, R_{7}$ and $R_{4}$ make up the supply list, and the solution is stored in the table consensus of the database. All this information is sent to experts by means of their mobile phones (Figure 16).

\section{Discussion: Drawbacks And Advantages}

In this section we point out some drawbacks and advantages of the implemented mobile DSS.

- Drawbacks:

We find the following drawbacks of our system:

1) To take part in the GDM process the users need a last generation mobile device to install the MDSS and this could be very expensive for them.

2) The user interfaces have to be easy and very simple because the mobile device screen is very small.

3) This mobile DSSs prototype can only be applied in numerical decision contexts, and it would be desirable to use other more flexible frameworks, such as linguistic contexts.
4) Some studies on the incorporation of consistency measures and dealing with missing values it would be desirable.

- Advantages:

On the other hand, we find the following advantages:

1) This mobile DSS allows to develop a distributed GDM process because the experts do not have to gather together to discuss the problem to solve.

2) This mobile DSS improves the speed of the classical DSSs because the experts receive and send the information using their mobile devices, which are carried at all times.

3) This mobile DSS provides a higher flexibility degree in the representation of preferences because the experts can use different preference representations formats to express their opinions. In such a way, we allow experts to provide their preferences anywhere, anytime and in multiple formats.

4) This mobile DSS incorporates a feedback mechanism that provides linguistic recommendations to the experts to quickly obtain a high consensus degree.

5) This mobile DSS allows to address large sets of alternatives in decision problems because incorporates the management of dynamic sets of alternatives.

\section{CONCLUDING REMARKS}

We have presented a prototype of Mobile DSS for GDM problems based on dynamic decision environments which incorporates a new tool to manage dynamic inputs and outputs of alternatives in the set of solution alternatives throughout the decision process. The prototype uses the advantages of mobile Internet technologies to improve the user satisfaction with the decision process and develop decision processes at anytime and anywhere. We have used mobile phones as the device used by the experts to send their preferences but the structure of the prototype is designed to use any other mobile device, such as PDAs. The prototype can be used with four different formats to represent the preferences in the best way according to the kind of problem and the experts' knowledge level.

\section{ACKNOWLEDGMENT}

This work has been supported by the Research Projects TIN2007-61079 and TIC-05299.

\section{REFERENCES}

[1] J. Kacprzyk and M. Fedrizzi, Multiperson decision making models using fuzzy sets and possibility theory. Dordrecht: Kluwer Academic Publishers, 1990.

[2] M. Roubens, "Fuzzy sets and decision analysis," Fuzzy Sets and Systems, vol. 90, no. 2, pp. 199-206, 1997.

[3] D. Ben-Arieh and Z. Chen, "Linguistic-labels aggregation and consensus measure for autocratic decision making using group recommendations," IEEE Transactions on Systems, Man, and Cybernetics. Part A: Systems and Humans, vol. 36, no. 3, pp. 558-568, 2006.

[4] D. Ben-Arieh, T. Easton, and B. Evans, "Minimum cost consensus with quadratic cost functions," IEEE Transactions on Systems, Man and Cybernetics Part A: Systems and Humans, vol. 39, no. 1, pp. 210-217, 2009.

[5] C. Dae-Young, "Aggregation of preferences based on fsam in gdss," IEEE Transactions on Systems, Man and Cybernetics Part A: Systems and Humans, vol. 38, no. 1, pp. 2-8, 2008. 
[6] J. Fodors and M. Roubens, Fuzzy preference modelling and multicriteria decision support. Dordrecht: Kluwer Academic Publishers, 1994.

[7] F. Herrera, E. Herrera-Viedma, and J. Verdegay, "A sequential selection process in group decision making with a linguistic assessment approach," Information Sciences, vol. 85, no. 4, pp. 223-239, 1995.

[8] _ _A model of consensus in group decision making under linguistic assessments," Fuzzy Sets and Systems, vol. 78, no. 1, pp. 73-87, 1996.

[9] F. Herrera, E. Herrera-Viedma, and L. Martinez, "A fuzzy linguistic methodology to deal with unbalanced linguistic term sets," IEEE Transactions on Fuzzy Systems, vol. 16, no. 2, pp. 354-370, 2008.

[10] L. Kitainick, Fuzzy Decision Procedures with Binary Relations, Towards an Unified Theory. Dordrecht: Kluwer Academic Publishers, 1993.

[11] C. Kobashikawa, Y. Hatakeyama, F. Dong, and K. Hirota, "Fuzzy algorithm for group decision making with participants having finite discriminating abilities," IEEE Transactions on Systems, Man and Cybernetics Part A: Systems and Humans, vol. 39, no. 1, pp. 86-95, 2009.

[12] J. Aronson, T. Liang, and E. Turban, Decision support systems and intelligent systems. Upper Saddle River: Pearson, 2005.

[13] F. Burstein and C. Holsapple, Handbook on Decision Support Systems. Springer, 2008.

[14] S. French and M. Turoff, "Decision support systems," Communications of the ACM, vol. 50, no. 3, pp. 39-40, 2007.

[15] J. Muntermann, Event-Driven mobile Finantial Information Services: Mobile notification and Decision Support for private investors. DUV, 2008.

[16] J. Schiller, Mobile Communications (2nd Edition). Addison Wesley, 2003.

[17] J. Katz, Handbook of Mobile Communication Studies. The MIT Press, 2008.

[18] F. Cabrerizo, S. Alonso, and E. Herrera-Viedma, "A consensus model for group decision making problems with unbalanced fuzzy linguistic information," International Journal of Information Technology \& Decision Making, vol. 8, no. 1, pp. 109-131, 2009.

[19] G. Buyukozkan, O. Feyzioglu, and D. Ruan, "Fuzzy group decisionmaking to multiple preference formats in quality function deployment," Computers In Industry, vol. 58, no. 5, pp. 392-402, 2007.

[20] F. Chiclana, F. Herrera, and E. Herrera-Viedma, "Integrating three representation models in fuzzy multipurpose decision making based on fuzzy preference relations," Fuzzy Sets and Systems, vol. 97, no. 1, pp. 33-48, 1998.

[21] _ - "Integrating multiplicative preference relations in a multiplicative decision making model based on fuzzy preference relations," Fuzzy Sets and Systems, vol. 122, no. 2, pp. 277-291, 2001.

[22] J. Ma, Z. Fan, and Y. Jiang, "An optimization approach to multiperson decision making based on different formats of preference information," IEEE Transactions on Systems, Man and Cybernetics part A-Systems and Humans, vol. 36, no. 5, pp. 876-889, 2006.

[23] Z. H. Xu, "Multiple-attribute group decision making with different formats of preference information on attributes," IEEE Transactions on Systems, Man and Cybernetics part B-Cybernetics, vol. 37, no. 6, pp. 1500-1511, 2007.

[24] Z. S. Xu and J. Chen, "Magdm linear-programming models with distinct uncertain preference structures," IEEE Transactions on Systems, Man and Cybernetics part B-Cybernetics, vol. 38, no. 5, pp. 1356-1370, 2008.

[25] J. Cowie and F. Burstein, "Quality of data model for supporting mobile decision making," Decision Support Systems, vol. 43, pp. 1675-1683, 2007.

[26] U. Varsney and R. Vetter, "Emerging mobile and wireless networks," Communications of the ACM, vol. 43, no. 6, pp. 73-81, 2000.

[27] T. Imielinski and B. Badrinath, "Mobile wireless computing: challenges in data management," Communications of the ACM, vol. 37, no. 10, pp. 18-28, 1994.

[28] W. Wen, Y. Chen, and H. Pao, "A mobile knowledge management decision support system for automatically conducting an electronic business," Knowledge-Based Systems, vol. 21, no. 7, 2008.

[29] A. Eren, A. Subasi, and O. Coskun, "A decision support system for telemedicine through the mobile telecommunications platform," Journal of Medical Systems, vol. 32, no. 1, 2008.

[30] F. Ricci and Q. Nguyen, "Acquiring and revising preferences in a critique-based mobile recommender system," IEEE Intelligent Systems, vol. 22, no. 3, 2007.

[31] E. Triantaphyllou, Multi-criteria decision making methods: a comparative study. Dordrecht/Boston/London: Kluwer Academic Publishers, 2000.
[32] R. Yager, "On ordered weighted averaging aggregation operators in multicriteria decision making," IEEE Transactions on Systems, Man, and Cybernetics, vol. 18, no. 1, pp. 183-190, 1988.

[33] _ "Weighted maximum entropy owa aggregation with applications to decision making under risk," IEEE Transactions on Systems, Man, and Cybernetics part A-Systems and Humans, vol. 39, no. 3, pp. 555564, 2009.

[34] E. Herrera-Viedma, F. Herrera, and F. Chiclana, "A consensus model for multiperson decision making with different preference structures," IEEE Transactions on Systems, Man, and Cybernetics. Part A: Systems and Humans, vol. 32, no. 3, pp. 394-402, 2002.

[35] "Midp 2.0 specifications." [Online]. Available: http://java.sun.com/products/midp/

[36] "Java wireless toolkit." [Online]. Available: http://java.sun.com/products/sjwtoolkit/

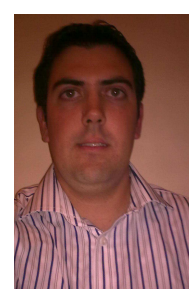

Ignacio Javier Pérez was born in Granada, on June 8, 1984. He received the M.Sc. and Master degrees in computer sciences from the University of Granada, Granada, Spain, in 2007 and 2008, respectively.

He is currently a research fellow in the Department of Computer Science and Artificial Intelligence, University of Granada. His current research interests focus on group decision making, decision support systems, consensus models, linguistic modeling, modeling situations with missing/incomplete information, aggregation of information, digital libraries and Web quality evaluation.

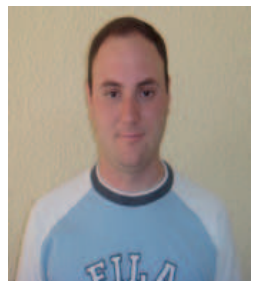

Francisco Javier Cabrerizo was born in Jódar, Spain, on February 8, 1983. He received the M.Sc. and $\mathrm{Ph} . \mathrm{D}$. degrees in computer sciences from the University of Granada, Granada, Spain, in 2006 and 2008, respectively.

$\mathrm{He}$ is currently a Professor in the Department of Software Engineering and Computer Systems, Distance Learning University of Spain (UNED), Madrid, Spain. He is a member of the Editorial Board of the Journal of Universal Computer Science. His current research interests focus on group decision making, decision support systems, consensus models, linguistic modeling, modeling situations with missing/incomplete information, aggregation of information, digital libraries, Web quality evaluation, and bibliometric measures.

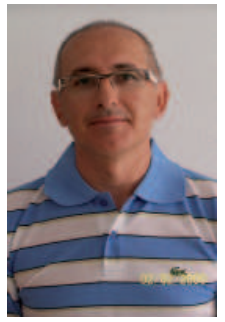

Enrique Herrera-Viedma was born in 1969. He received the M.Sc. and Ph.D. degrees in computer sciences from the University of Granada, Granada, Spain, in 1993 and 1996, respectively.

$\mathrm{He}$ is currently a Full Professor in the Department of Computer Science and Artificial Intelligence, University of Granada. He has authored or coauthored more than 80 papers published in international journals. His h-index is 24 and he is classed as one of the "Most Cited Scientists in Engineering" in the ISI Web of Science - Essential Science Indicators, i.e. he is in the top $1 \%$ of most cited scientists in the field of engineering. Six of his papers are classed as highly cited as well, being in the top $1 \%$ of most cited papers in its field. He is a member of the Editorial Board of the Fuzzy Sets and Systems, Soft Computing, and International Journal of Information Technology and Decision Making. His current research interests include group decision making, consensus models, linguistic modeling, aggregation of information, information retrieval, bibliometric measures, digital libraries, Web quality evaluation, and recommender systems. 